Supporting Information

\title{
Development of Icephilic ACTIVE Glycopeptides for Cryopreservation of Human Erythrocytes
}

Shuhui Gao, ${ }^{\dagger}$ Kongying Zhu,${ }^{\ddagger}$ Qifa Zhang, ${ }^{\dagger}$ Qingjing Niu,${ }^{\dagger}$ Jinghui Chong,${ }^{\S}$ Lixia Ren,${ }^{\dagger}$ and Xiaoyan Yuan ${ }^{*}, \dagger$

${ }^{\dagger}$ School of Materials Science and Engineering, Tianjin Key Laboratory of Composite and Functional Materials, Tianjin University, Tianjin 300350, China

\#Analysis and Measurement Center, Tianjin University, Tianjin 300072, China

${ }^{\S}$ Tianjin Blood Center, Tianjin 300110, China

"E-mail: yuanxy@tju.edu.cn 
Materials. $\quad \varepsilon$-Poly(L-lysine) $(\varepsilon-\mathrm{PL}$, average molecular weight $\sim 4000)$ was purchased from

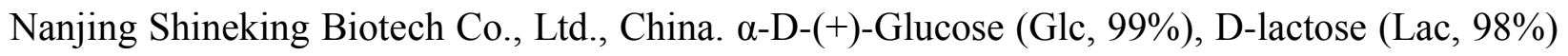
and $\alpha, \alpha$-trehalose (Tre, 99\% anhydrous) were provided by Beijing J\&K Scientific Co., Ltd., China. Maltose (Mal, BR 95\%) was obtained from Shanghai Yuanye Bio-Technology Co., Ltd., China. Maltotriose (Mal(III), 96\%) was provided by Shanghai Macklin Biochemical Co., Ltd., China. Sodium cyanoborohydride $\left(\mathrm{NaBH}_{3} \mathrm{CN}\right)$ was purchased from Shanghai Aladdin Biochemical Technology Co., Ltd., China. 1-Ethyl-3-(3-dimethylaminopropyl)carbodiimide hydrochloride $\quad($ EDCI), $\quad N$-Hydroxy $\quad$ succinimide $\quad$ (NHS), 4-(2-hydroxyethyl)piperazine-1-ethanesulfonic acid (HEPES, 99.5\%) and sucrose (Suc, 99\%) were obtained from Tianjin Heowns Biochemical Technology Co., Ltd., China. 1,2-Dioleoyl-sn-glycero-3-phosphocholine (DOPC) was purchased by Sigma-Aldrich. 5-Carboxyfluorescein (5-FAM, $\geq 98 \%$ ) was obtained by Shanghai D\&B Chemical Technology Co., Ltd., China. FITC-D-trehalose dihydrate (Tre-FITC) was provided by Xi'an Qiyue Biological Biotechnology Co., Ltd., China. N,N-Dimethylformamide (DMF, AR) was provided by Tianjin Jiangtian Chemical Technology Co., Ltd., China. Glacial acetic acid (GAA, $\geq 99.5 \%$ ) was purchased from Tianjin Kermel Chemical Reagent Co., Ltd., China. All chemicals were used as received without further purification. Enhanced adenosine triphosphate (ATP) Assay Kit and Detergent Compatible Bradford Protein Assay Kit were supplied by Beyotime Biotechnology, China. Human 2,3-diphosphoglycerate (2,3-DPG) ELISA Kit was purchased from Nanjing Herb-Source Bio-Technology, China. Methemoglobin (MetHb) Assay Kit was supplied by 
Nanjing Jiancheng Bioengineering Institute, China. FITC Annexin V Apoptosis Detection Kit I (BD Biosciences, USA) was used for phosphatidylserine (PS) exposure determination.

Synthesis. As shown in Scheme 1, sugar-grafted $\varepsilon$-poly(L-lysine) (PL-g-S), depicted as PL-g-Glc, PL-g-Lac, PL-g-Mal, PL-g-Mal(III) and PL-g-Tre were synthesized, respectively, according to the references. ${ }^{1-3}$ Glucose $(1.00 \mathrm{~g}, 5.47 \mathrm{mmol})$, lactose $(1.91 \mathrm{~g}, 5.47 \mathrm{mmol})$, maltose $(1.97 \mathrm{~g}, 5.47 \mathrm{mmol})$ or maltotriose $(2.87 \mathrm{~g}, 5.47 \mathrm{mmol})$ was vigorously mixed with $\varepsilon$-PL $(0.50 \mathrm{~g}$, $3.91 \mathrm{mmol})$ in a DMF/DI water/GAA $(6 / 3 / 1,40 \mathrm{~mL})$ mixture and stirred at $55^{\circ} \mathrm{C}$ for $1 \mathrm{~h}$. Then $\mathrm{NaBH}_{3} \mathrm{CN}(0.344 \mathrm{~g}, 5.47 \mathrm{mmol})$ was added in batches. After stirring at $55^{\circ} \mathrm{C}$ for total $72 \mathrm{~h}$, the crude product was purified via dialysis (MWCO 3500 Dalton) against DI water for 3 days and lyophilized. Coincidentally, EDCI (0.24 g, $1.25 \mathrm{mmol})$ and NHS (0.14g, $1.25 \mathrm{mmol})$ were stirred in DI water $(5 \mathrm{~mL})$ at room temperature for $30 \mathrm{~min}$ with carboxylated trehalose $(0.55 \mathrm{~g}, 1.25$ mmol), which was synthesized according to the previous publication, ${ }^{3}$ before addition of $\varepsilon$-PL (0.11 g, $0.89 \mathrm{mmol})$ in DMSO $(5 \mathrm{~mL})$. The mixture was stirred at R.T. for 3 days and followed the aforementioned post-procedure.

Characterizations. Chemical structures of the synthesized glycopeptides, PL-g-Glc, PL-g-Lac, PL-g-Mal, PL-g-Mal(III) and PL-g-Tre were verified by a ${ }^{1} \mathrm{H}$ NMR spectrometer (AVANCE III TM HD 400 MHz NanoBAY, Bruker, Germany) and a FTIR spectrometer (IRtracer100, Shimadzu, Japan) using $\mathrm{KBr}$ pellet technique ranging from $4000 \mathrm{~cm}^{-1}$ to $400 \mathrm{~cm}^{-1}$. The molecular weight of PL-g-S was assessed by an aqueous gel permeation chromatography (GPC, Viscotek, UK) system with HAc/NaAc (0.5M, pH 4.5) buffer as the eluate, and calibrated 
with poly(ethylene glycol) standards. The hydrodynamic diameter and Zeta potential of PL- $g$-S with a concentration of $1.0 \mathrm{mg} \mathrm{mL}^{-1}$ were evidenced by a dynamic light scatterer (DLS, Zetasizer Nano ZS90, Malvern, UK) after stabilizing at room temperature for $48 \mathrm{~h}$ before tests.

Analysis of Chemical Structure of the Glycopeptides. The ${ }^{1} \mathrm{H}$ NMR and FTIR spectra of the glycopeptides confirmed the chemical structures and all as-expected glycosidic bonds occurred. As demonstrated in Figure $\mathrm{S} 1(\mathrm{a})$, original $-\mathrm{CH}_{2}-$ and $-\mathrm{OH}$ - of sugar moieties were mainly located at $\delta$ 3.28-3.99 ppm. Afterwards, newly generated $-\mathrm{NH}-\mathrm{CH}_{2}$ - group in as-synthesized glycopeptides, PL-g-Glc, PL-g-Lac, PL-g-Mal or PL-g-Mal(III), belongs to $\delta$ 2.61-2.72 ppm, $\delta 2.63-2.88 \mathrm{ppm}, \delta 2.72 \mathrm{ppm}$ or $\delta 2.73 \mathrm{ppm}$, respectively, which was integrated with respect to $-\mathrm{CH}\left(\mathrm{NH}_{2}\right)-\mathrm{CH}_{2}-\mathrm{CH}_{2}$ - in $\varepsilon$-PL backbone at around $\delta 1.33 \mathrm{ppm}$ to estimate the grafting degree. From the result of ${ }^{1} \mathrm{H}$ NMR spectra, averaged 28, 28, 27 or 27 molar amounts of mono/oligo-saccharides was successfully grafted onto a $\varepsilon$-PL backbone abundant in amino groups ( $c a .31-\mathrm{NH}_{2}$ for each). About 89.3, 90.9, 86.8 and 86.6\% of grafting ratios for PL-g-Glc, PL-g-Lac, PL-g-Mal and PL-g-Mal(III), respectively, were successfully obtained as listed in (Table 1).

Coincidently, the proton integration ratio of $-\mathrm{CH}$ - at $1,1^{\prime}$ position of trehalose at $5.16 \mathrm{ppm}$ and $-\mathrm{NH}-\mathrm{CH}_{2}-\mathrm{CH}_{2-}$ of $\varepsilon-\mathrm{PL}$ backbone at around $\delta 3.20 \mathrm{ppm}$ proceeded via adopting the same calculated approach, as depicted in Figure S1(b) of the ${ }^{1} \mathrm{H}$ NMR spectra. Although the feeding ratio of $\left[\mathrm{NH}_{2}\right]$ and $[\mathrm{COOH}]$ was performed as a molar ratio of 1:1.4 theoretically, the grafting ratio of PL-g-Tre was $42.2 \%$, much lower than those of others. Averaged 13 molar amounts of 
trehalose were successfully grafted onto a $\varepsilon$-PL backbone. In particular, low grafting ratio of PL- $g$-Tre was more likely to ascribe to the low efficient amidation reaction with side effects.
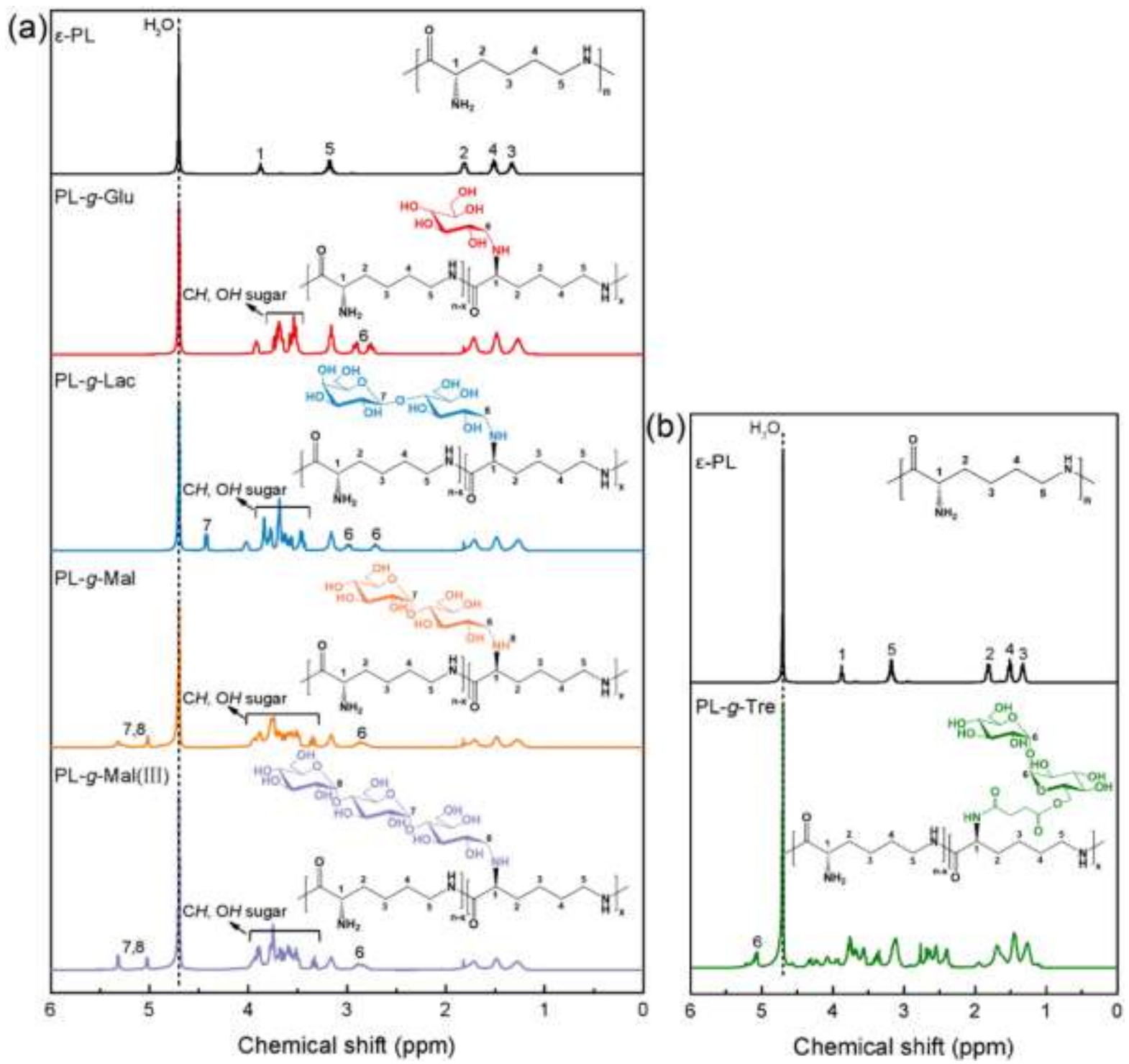

Figure S1. $\quad{ }^{1} \mathrm{H}$ NMR spectra of $\varepsilon$-PL, PL- $g$-Glc, PL-g-Lac, PL-g-Mal and PL-g-Mal(III) (a), as well as $\varepsilon$-PL and PL- $g$-Tre (b). 
The chemical structure of the glycopeptides was also verified by FTIR spectra as shown in Figure S2. The vibration absorption bands of amido bond in accordance with amide I and amide II at $1673 \mathrm{~cm}^{-1}$ and $1565 \mathrm{~cm}^{-1}$, respectively, existed on both $\varepsilon$-PL and PL-g-S backbones, and $\mathrm{C}-\mathrm{H}$ bond signal of $-\mathrm{CH}_{2}$ - groups for $\varepsilon-\mathrm{PL}$ whose stretching vibration characteristic peak at 2936 $\mathrm{cm}^{-1}$ was apparently weaker than that for PL-g-S which encompasses $-\mathrm{CH}_{2} \mathrm{OH}$ groups of grafted mono/oligo- saccharide. Moreover, exclusive glycosidic bonds for each oligosaccharide appeared in all the curves of the samples. To be specific, the characteristic absorption peak at $889 \mathrm{~cm}^{-1}$ belonged to $\beta$-glycosidic bond of lactose, and $\alpha$-glycosidic bonds of maltose/maltotriose and trehalose were assigned at $927 / 849 \mathrm{~cm}^{-1}$ and $870 / 806 \mathrm{~cm}^{-1}$, respectively. In particular, residual ester groups whose stretching vibration absorption peak at $1729 \mathrm{~cm}^{-1}$ appeared for successful grafting of trehalose onto PL-g-Tre. 


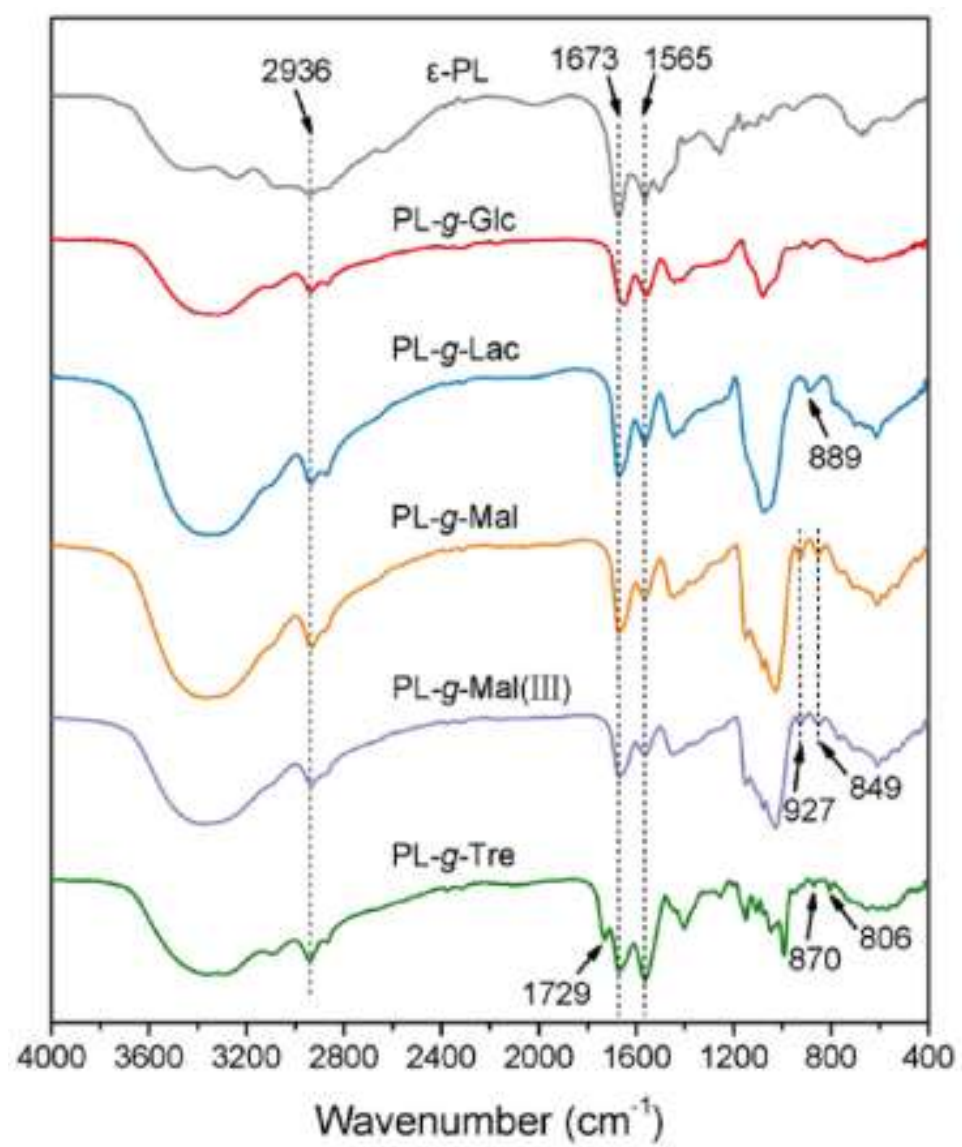

Figure S2. FTIR spectra of $\varepsilon$-PL, PL-g-Glc, PL-g-Lac, PL- $g$-Mal, PL-g-Mal(III) and PL- $g$-Tre. 
Confocal Microscopy. 5-Carboxyfluorescein (5-FAM) labeled glycopeptides (PL-g-S-g-FAM) were prepared through amidation. ${ }^{4}$ Human erythrocytes $(\sim 10 \%$ hematocrit $)$ were incubated with $1.0 \mathrm{mg} \mathrm{mL}^{-1} \mathrm{PL}-g$-S-g-FAM solution containing $0.36 \mathrm{M}$ trehalose in $1 \times$ HEPES at $4{ }^{\circ} \mathrm{C}$ for $7 \mathrm{~h}$. After diluting 10 times by $1 \times$ HEPES, a $10 \mu \mathrm{L}$ aliquot of the $\mathrm{hRBC}$ suspensions after washing 3 times with $1 \times$ HEPES was transferred to a glass slide, on which a cover slip was placed. Cells were imaged at $60 \times$ magnification on a confocal laser scanning microscope (CLSM, A1R+, Nikon, Japan) with an excitation wavelength of 5-FAM at $488 \mathrm{~nm}$.

Cytotoxicity Test. Alamar Blue assay was performed to assess the inherent toxicity of the synthesized glycopeptides against L929 fibroblasts. ${ }^{5,6}$ Briefly, cells were seeded in 96-well microplates at a density of $1 \times 10^{4}$ cells per well in $100 \mu \mathrm{L}$ high-glucose DMEM complex culture medium containing $10 \mathrm{vol} . \%$ fetal bovine serum and $1 \mathrm{vol} \%$ penicillin-streptomycin. After incubation at $37{ }^{\circ} \mathrm{C}, 5 \% \mathrm{CO}_{2}$ for $24 \mathrm{~h}$, original culture medium was replaced by fresh one containing synthesized glycopeptides at specific concentrations for another $24 \mathrm{~h}$. Then, cells were washed with $1 \times$ PBS and incubated further with Alamar Blue solution $(100 \mu \mathrm{L}, 1: 9$ diluted in pure DMEM culture medium) away from light for $4 \mathrm{~h}$. Fluorescence intensity $(I)$ of the mixture was examined at the excitation wavelength of $525 \mathrm{~nm}$ and emission wavelength of 590 $\mathrm{nm}$ using a spectrophotometer (Synergy H1, BioTek, USA). Cells incubation lack of as-synthesized glycopeptides acted as controls, while the pure Alamar Blue solution absence of neither L929 cells nor as-synthesized glycopeptides was credited as blanks. Relative cell viability was proposed in accordance with the following equation (S1): 


$$
\text { Relative cell viability }(\%)=\frac{I_{590 \mathrm{~nm}, \text { Sample }}-I_{590 \mathrm{~nm}, \text { Blank }}}{I_{590 \mathrm{~nm}, \text { Control }}-I_{590 \mathrm{~nm}, \text { Blank }}} \times 100
$$

Determination of hRBCs Function after Cryostorage. Incubation of the samples ( 10\% hematocrit) was carried out in the $1.0 \mathrm{mg} \mathrm{mL}^{-1}$ glycopeptide solutions containing $0.36 \mathrm{M}$ trehalose in $1 \times$ HEPES at $4{ }^{\circ} \mathrm{C}$ for $7 \mathrm{~h}$, and subsequent cryopreservation at $-196{ }^{\circ} \mathrm{C}$. After washed 3 times with $1 \times \mathrm{PBS}$, the suspended erythrocytes before and after cryopreservation were employed for the function measurements by using the relevant diagnostics assay kits listed in the Supporting Information, including adenosine triphosphate (ATP), 2,3-diphosphoglycerate (2,3-DPG), methemoglobin (MetHb), phosphatidylserine (PS) exposure.

Icephilicity Tests. The performance of the synthesized glycopeptides against ice growth was evaluated by IRI, differential scanning calorimetry (DSC), Raman spectroscopy and dynamic ice shaping (DIS). The IRI activity of the synthesized glycopeptides was conducted according to a "splat cooling" assay. ${ }^{7,8}$ The PL- $g$-S solution with or without $0.36 \mathrm{M}$ trehalose in $1 \times$ HEPES at specific concentration of $1.0 \sim 8.0 \mathrm{mg} \mathrm{mL}^{-1}$ was tested by using $1 \times$ HEPES as an individual control. A $10 \mu \mathrm{L}$ droplet of the prepared PL-g-S solution was dropped from a $1.5 \mathrm{~m}$ height through a micropipette onto the surface of a precooled aluminium plate controlled at approximately $-80{ }^{\circ} \mathrm{C}$, on which a tailored crucible was placed. The droplet frozen promptly in the form of a thin film of ice with presumably $1 \mathrm{~cm}$ in diameter and was transferred onto a cryostage (THMS600, Linkam, UK) which was increased at a heating rate of $25{ }^{\circ} \mathrm{C} \mathrm{min}{ }^{-1}$ and held at $-8{ }^{\circ} \mathrm{C}$ for annealing. After a period of $35 \mathrm{~min}$, ice recrystallization of this wafer was photographed using an optical microscope (TI-S, Nikon, Japan) at 5 different locations, with the 
area of ten larger crystals in each image being quantified by ImageJ software, and the average of which is ultimately defined as mean largest grain size (MLGS) to evaluate the IRI activity.

DSC analysis was performed using a Q2000 differential calorimeter (TA Instruments, USA). ${ }^{9} 10$ Nanoscale liposomes of 1,2-dioleoyl-sn-glycero-3-phosphocholine (DOPC, $4 \mathrm{mM}$ ) were formed by ultrasonically dispersing in $1 \times$ HEPES, accompanied by 5 freeze-thaw cycles in $20 \mathrm{~min}$. Then, the dispersions encompassed of $1.0 \mathrm{mg} \mathrm{mL}^{-1} \mathrm{PL}-\mathrm{g}$-Mal, $0.36 \mathrm{M}$ trehalose or $1 \mathrm{mM}$ DOPC were tested. A $10 \mu \mathrm{L}$ aliquot of the dispersions was added in a liquid aluminum pan, keeping constant weight for 7 days before tests. The DSC measurements were carried out from $20{ }^{\circ} \mathrm{C}$ to $-50{ }^{\circ} \mathrm{C}$ at a cooling rate of $10{ }^{\circ} \mathrm{C} \min ^{-1}$, and kept at $-50{ }^{\circ} \mathrm{C}$ for $10 \mathrm{~min}$, followed by a slow rise to $20{ }^{\circ} \mathrm{C}$ at $2{ }^{\circ} \mathrm{C} \min ^{-1}$. Microscopic laser Raman spectroscopy of above dispersed samples in $30 \mu \mathrm{L}$ aliquot was also measured (DXR Thermo Fisher, USA). ${ }^{11-13}$ The Raman analysis was performed from $20{ }^{\circ} \mathrm{C}$ to $-20{ }^{\circ} \mathrm{C}$ at a cooling rate of $2{ }^{\circ} \mathrm{C} \min ^{-1}$, followed by a rapid fall to $-50{ }^{\circ} \mathrm{C}$ at $10{ }^{\circ} \mathrm{C} \min ^{-1}$. Samples were controlled at $20{ }^{\circ} \mathrm{C}, 0{ }^{\circ} \mathrm{C},-20{ }^{\circ} \mathrm{C}$ and $-50{ }^{\circ} \mathrm{C}$ for each 5 min, respectively, to obtain the Raman spectra.

DIS measurements were conducted using a nanoliter osmometer (Otago Osmometers Ltd., Dunedin, New Zealand) with a cooling rate of $0.01{ }^{\circ} \mathrm{C} / \mathrm{s}$ according to the previous testing system and procedure reported elsewhere. ${ }^{14,15}$ Growth processes of the single ice crystal was recorded by a high-speed camera (Phantom V7.3), and the growth rate of ice crystals $(\gamma)$ was obtained from the recorded images via dividing the time span $(\Delta T)$. 
Morphological Observation. Before and after incubation or cryopreservation, the suspended erythrocytes were fixed by $2.5 \%$ glutaraldehyde over $3 \mathrm{~h}$ at room temperature, accompanied by $1 \times$ PBS washing and dehydration with graded ethanol $(30,40,50,70,80,85,90$, 95 and $100 \%$ ) each for $5 \mathrm{~min}$. Then, the cells were observed under a scanning electron microscope (SEM, S-4800, Hitachi, Japan) after sputtered with gold. ${ }^{16}$ According to the references, ${ }^{17,18}$ erythrocytes were also imaged using a scanning probe atomic force microscope (AFM, CSPM5500A, Guangzhou, China) using a silicon tip with a resonance frequency of 300 $\mathrm{kHz}$, spring constant of $40 \mathrm{~N} \mathrm{~m}^{-1}$ and tapping amplitude of $1-2 \mathrm{~V}$ at room temperature in a tapping mode. Prior to imaging, the cells were subjected to incubation with $1.0 \mathrm{mg} \mathrm{mL}-1$ PL- $g$-Mal or $\varepsilon$-PL in $1 \times$ HEPES at $4{ }^{\circ} \mathrm{C}$ for $7 \mathrm{~h}$, and subsequently immobilized onto polylysine-coated microscope slides, fixed in 1\% glutaraldehyde at room temperature for $10 \mathrm{~min}$. The cells were further washed with $1 \times \mathrm{PBS}$ for 3 times and air dried before AFM measurement. The scanning scale is $350 \mathrm{~nm} \times 350 \mathrm{~nm}$ and the images are analyzed by Imager software. 

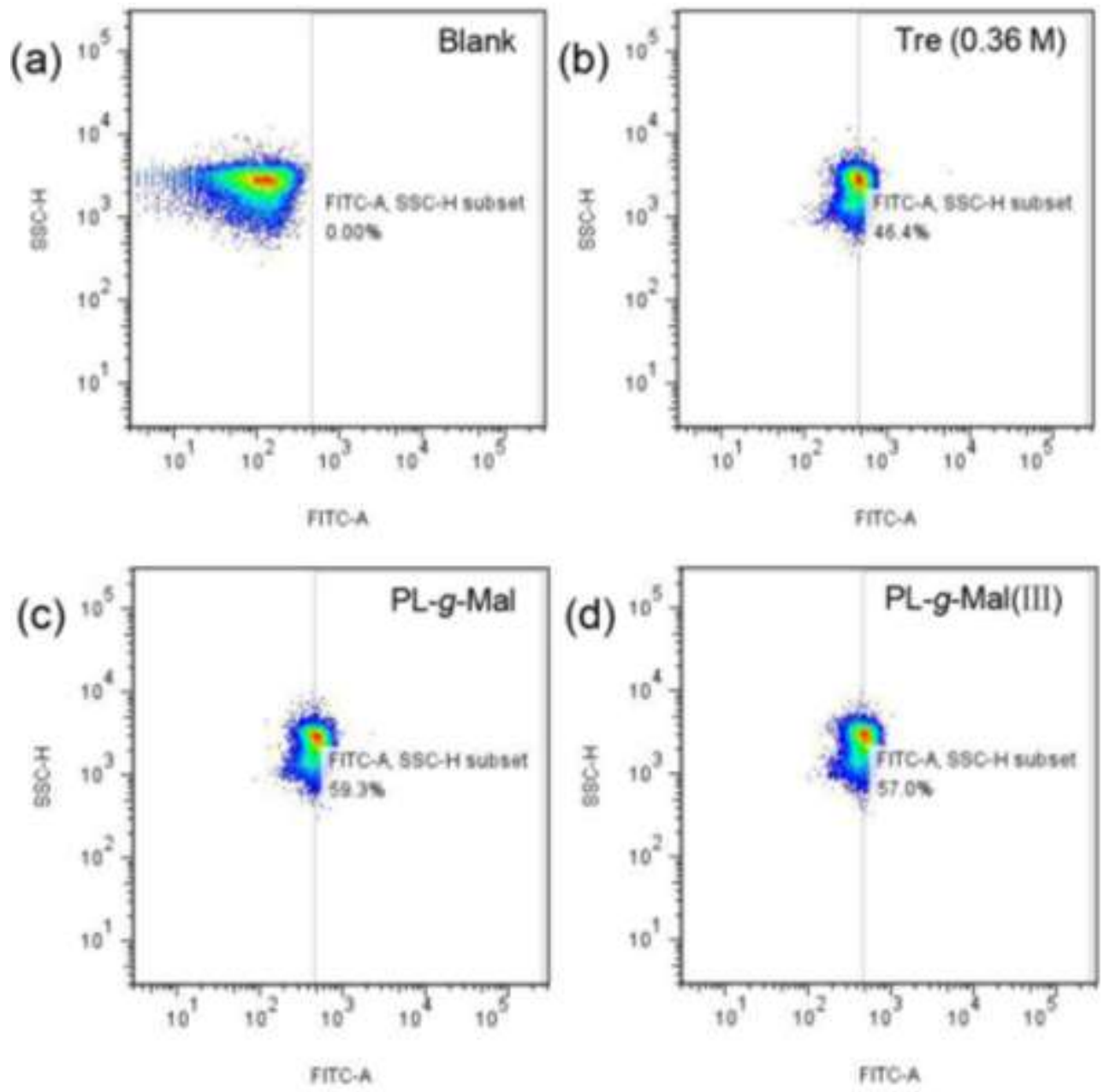

Figure S3. Scatter images of fresh human erythrocytes (a) incubated by $0.36 \mathrm{M}$ trehalose (b), Tre/PL-g-Mal (c) and Tre/PL-g-Mal(III) (d) measured by flow cytometry. 
Hemolytic activity and Cryopreservation.

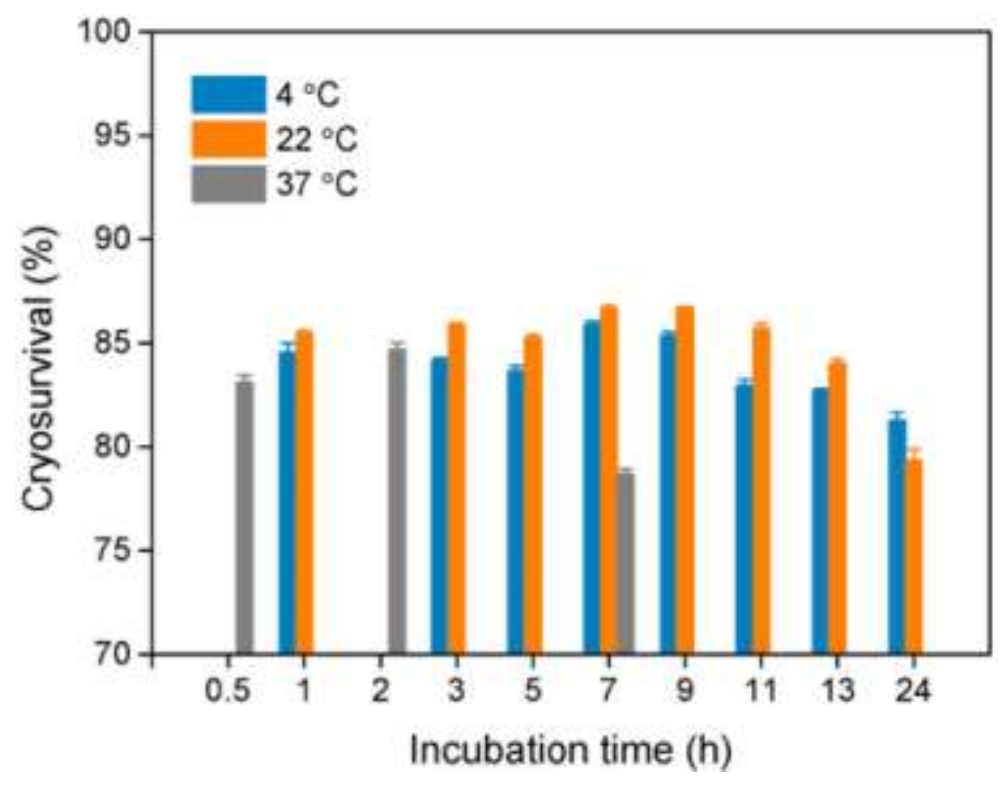

Figure S4. Effect of the incubation temperature and incubation time in $0.36 \mathrm{M}$ trehalose on cryosurvivial of hRBC.

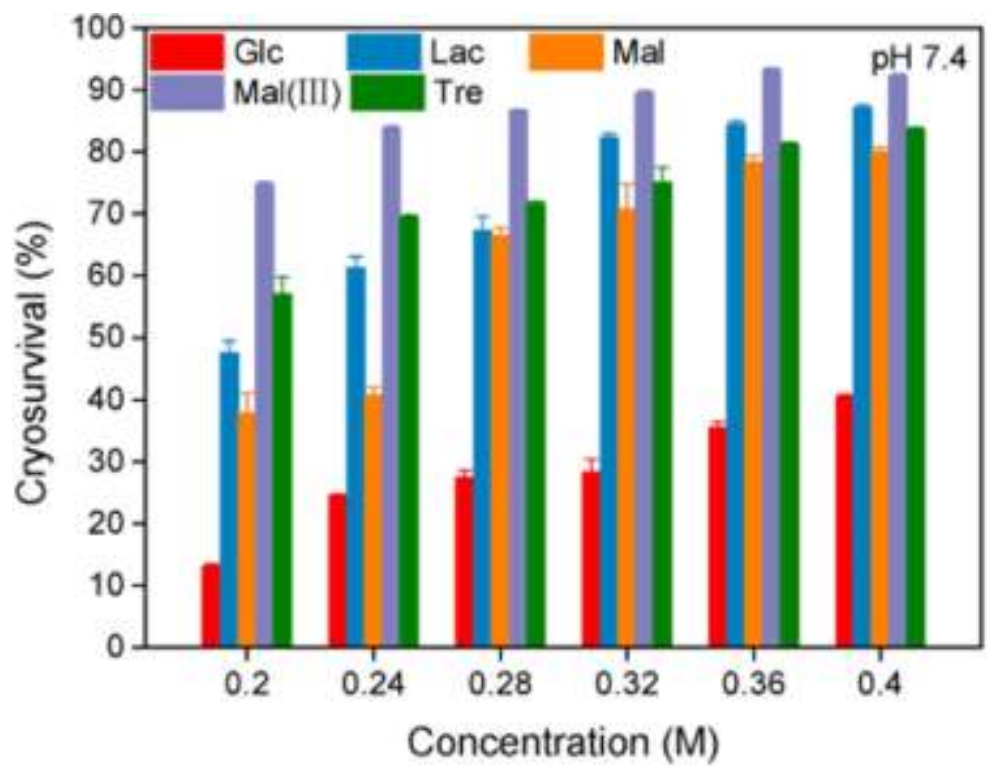

Figure S5. Cryosurvival variation of mono-/oligo-Saccharides containing glucose (Glc), lactose (Lac), maltose (Mal), maltotriose (Mal(III)) or trehalose (Tre) with different concentration at $\mathrm{pH} 7.4$. 


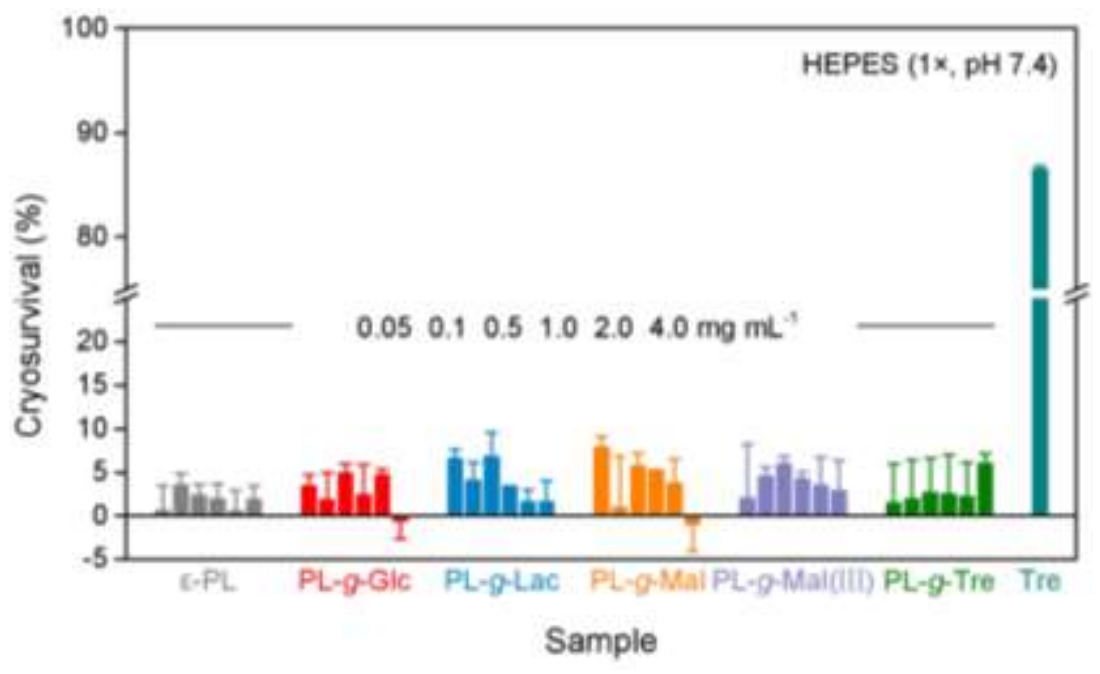

Figure S6. Cryosurvival variation of PL-g-S with different concentrations at pH 7.4.
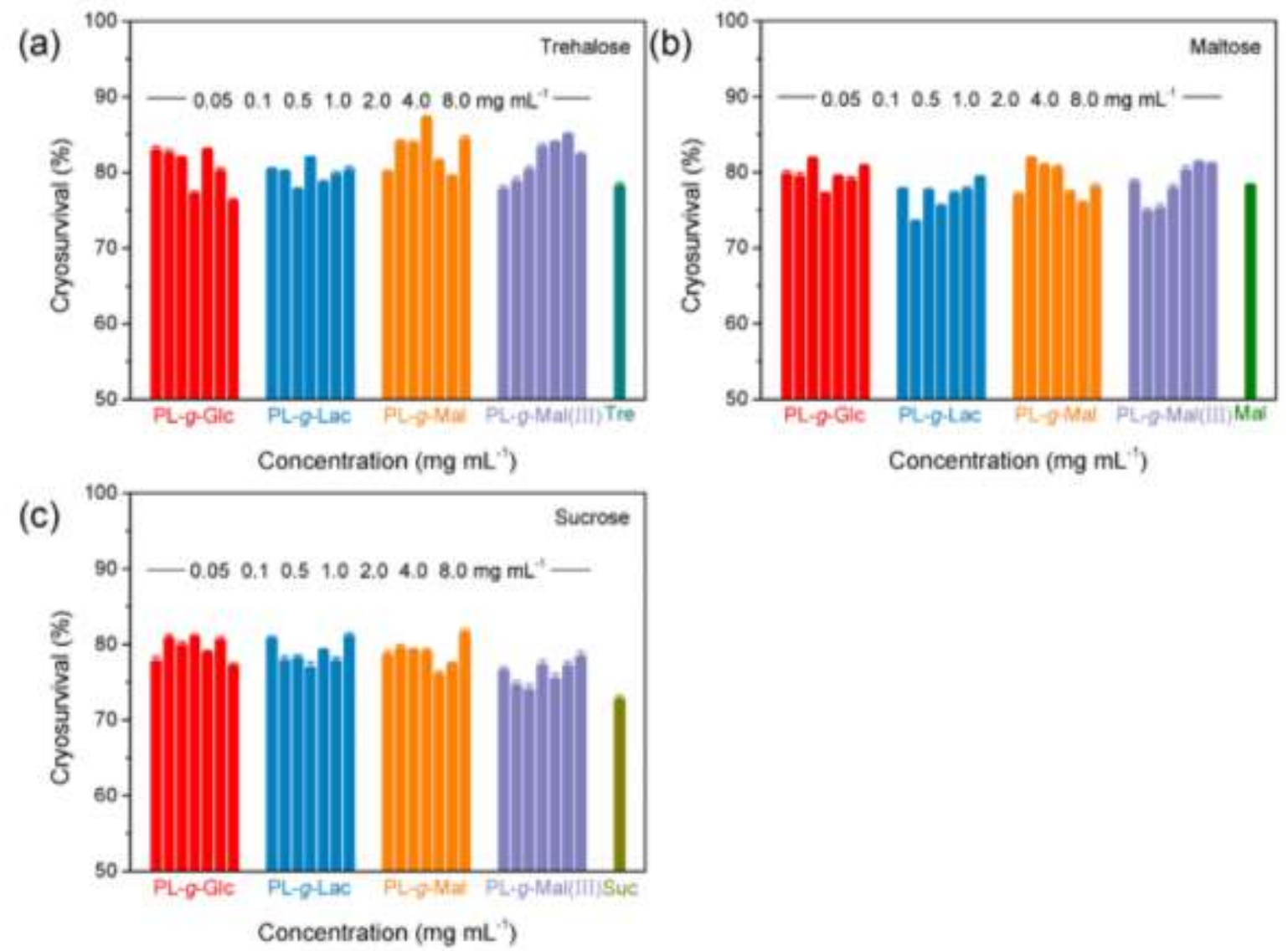

Figure S7. Cryosurvival variation of PL-g-S with different concentrations at $\mathrm{pH} 7.4$ accompanied by $0.36 \mathrm{M}$ trehalose (a), maltose (b) and sucrose (c), respectively. 
For the sake of investigating the relationship of haemoglobin absorbance and erythrocyte concentration, the washed erythrocyte pellet was resuspended in $1 \times \mathrm{PBS}$ at $1 / 1(v / v)$ ratio and the erythrocyte concentration was counted by an automated hematology analyzer (Bowlinman BM830, Beijing, China). Then, 25, 50, 75, 100, 150 and $200 \mu \mathrm{L}$ of cell suspensions were lysed in DI water to each constant of $1.2 \mathrm{~mL}$ and equilibrated at room temperature for $10 \mathrm{~min}$, accompanied by determination of haemoglogin absorbance at $541 \mathrm{~nm}$.

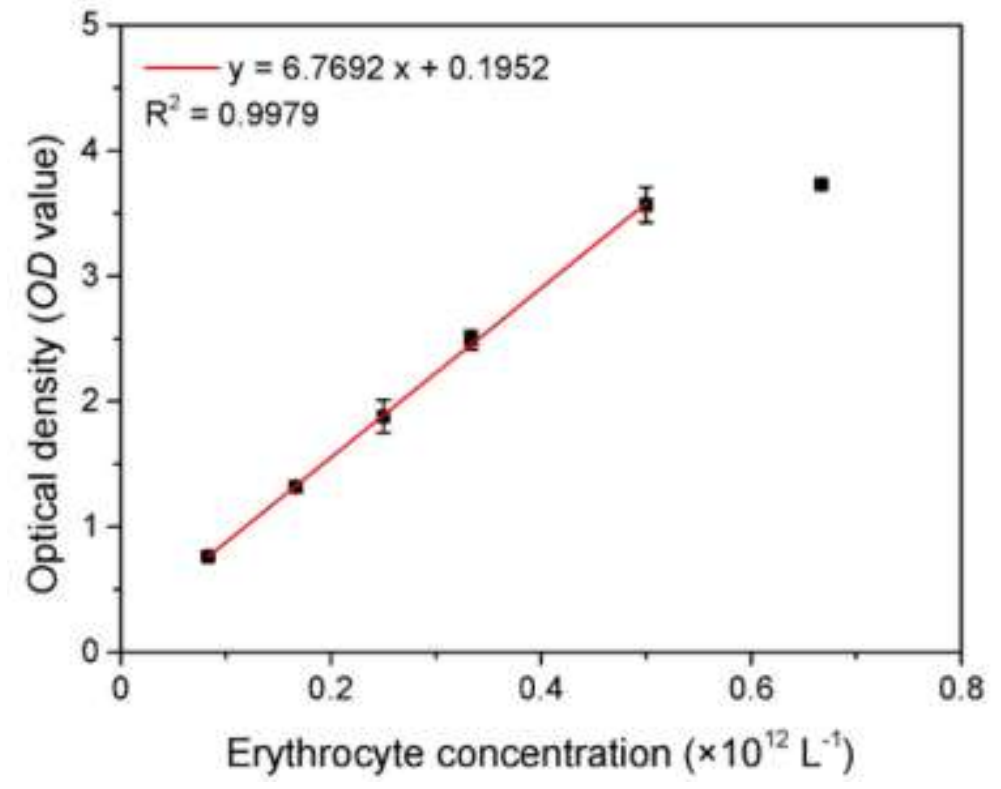

Figure S8. The relationship between $O D$ value and erythrocyte concentration measured by microplate reader. 

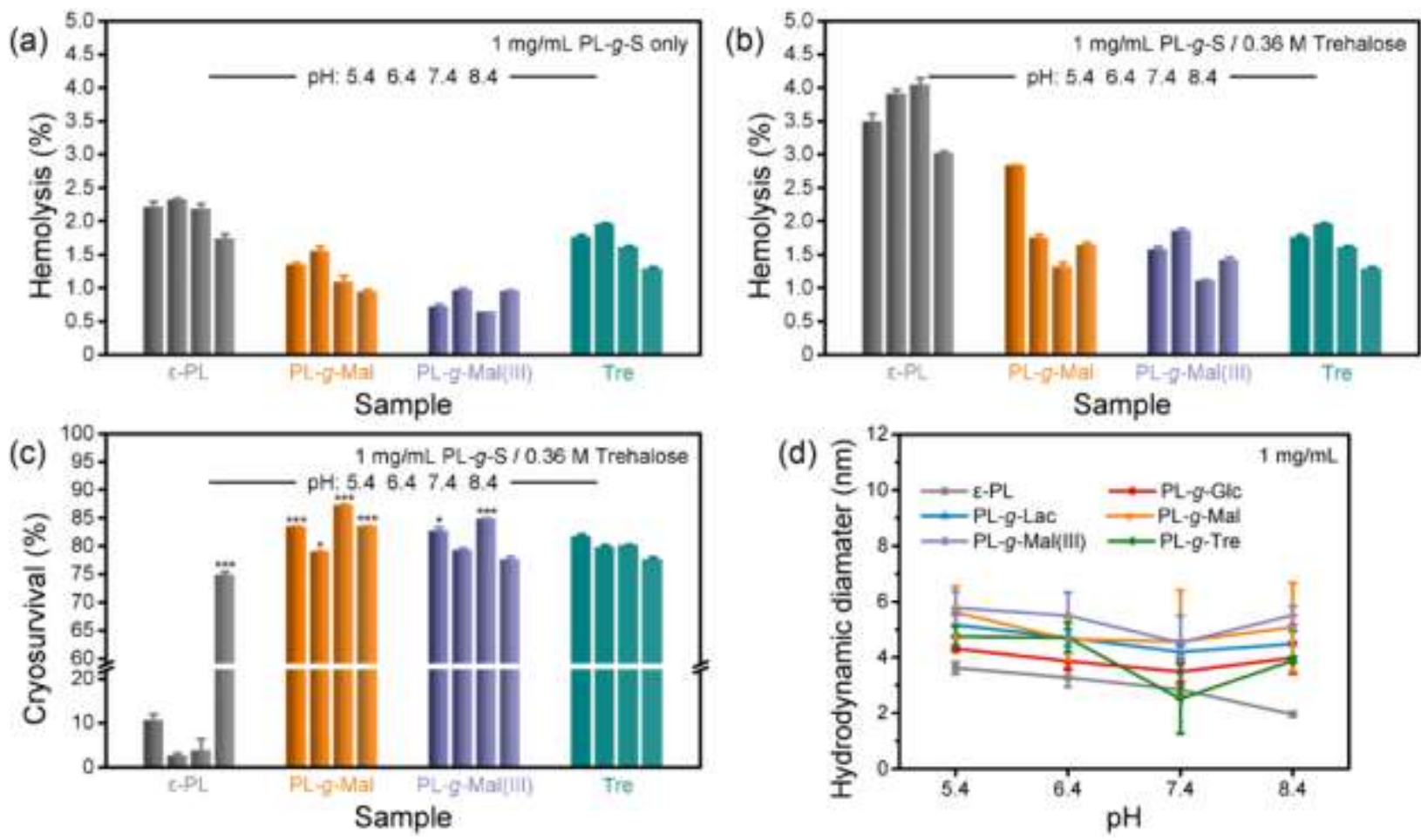

Figure S9. Hemolysis (a,b) and cryosurvival variations (c) of human erythrocytes after incubation in PL-g-S solutions at $1 \mathrm{mg} / \mathrm{mL}$ (a) accompanied by $0.36 \mathrm{M}$ trehalose (b,c) as well as hydrodynamic diameter (d) of PL-g-S with different pHs. 


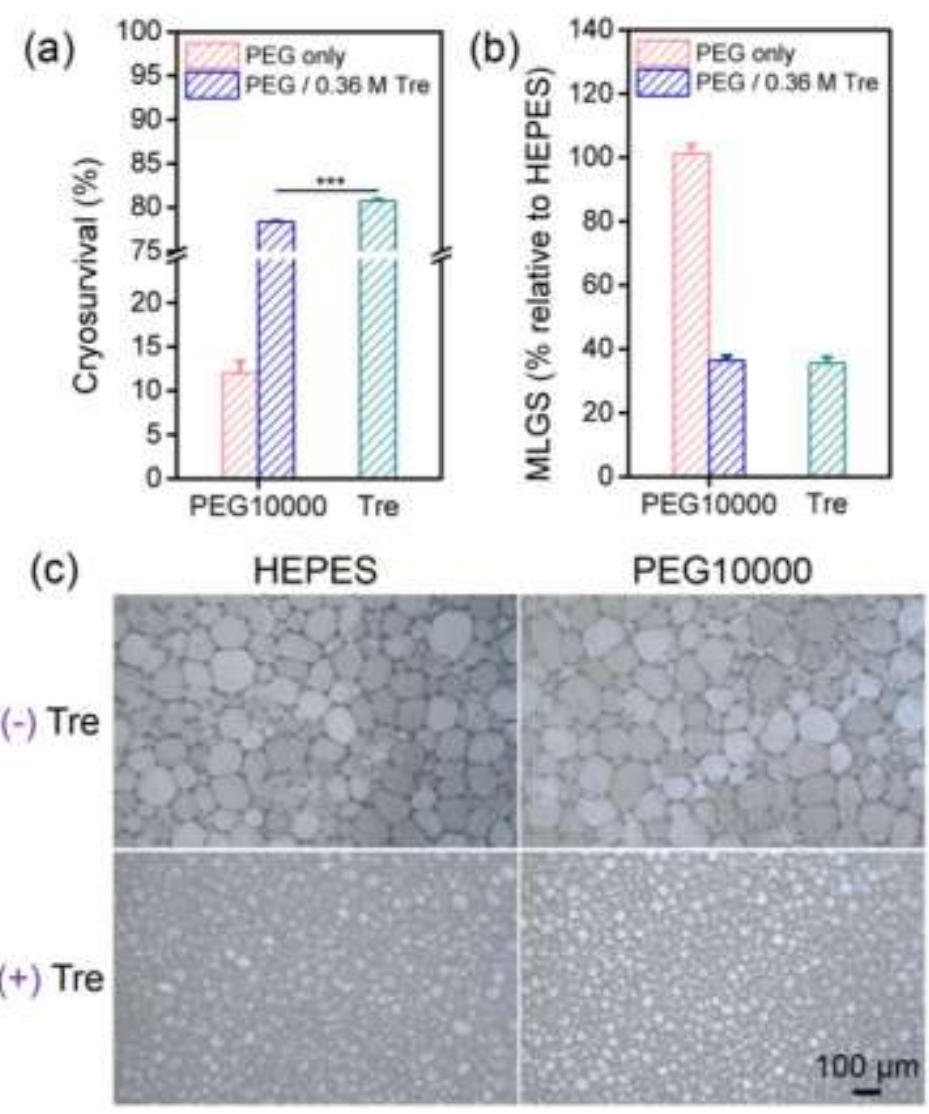

Figure S10. Cryosurvival variations (a) and the percentage of mean largest grain size (MLGS) relative to $1 \times$ HEPES (b) as well as the corresponding optical pictures of ice crystals (c) obtained from $1.0 \mathrm{mg} \mathrm{mL}^{-1}$ PEG (10 kDa) solution without or with $0.36 \mathrm{M}$ trehalose. 
Icephilicity and the corresponding cryogenic synergy with free trehalose. Freezing point is a kinetics-dependent quantity affected by many factors such as purity of aqueous medium and cooling rates. Given poor reproducibility of freezing point, equilibrium melting point $\left(T_{\mathrm{m}, \text { onset }}\right)$ mediated by thermodynamics is preferred on behalf of the initial melting temperature of ice, even though DSC cooling thermograms (Figure S9) showed the freezing point of PL- $g$-Mal/trehalose was observed to $-25.1{ }^{\circ} \mathrm{C}$, lower than that of each individual and even far below DI water.

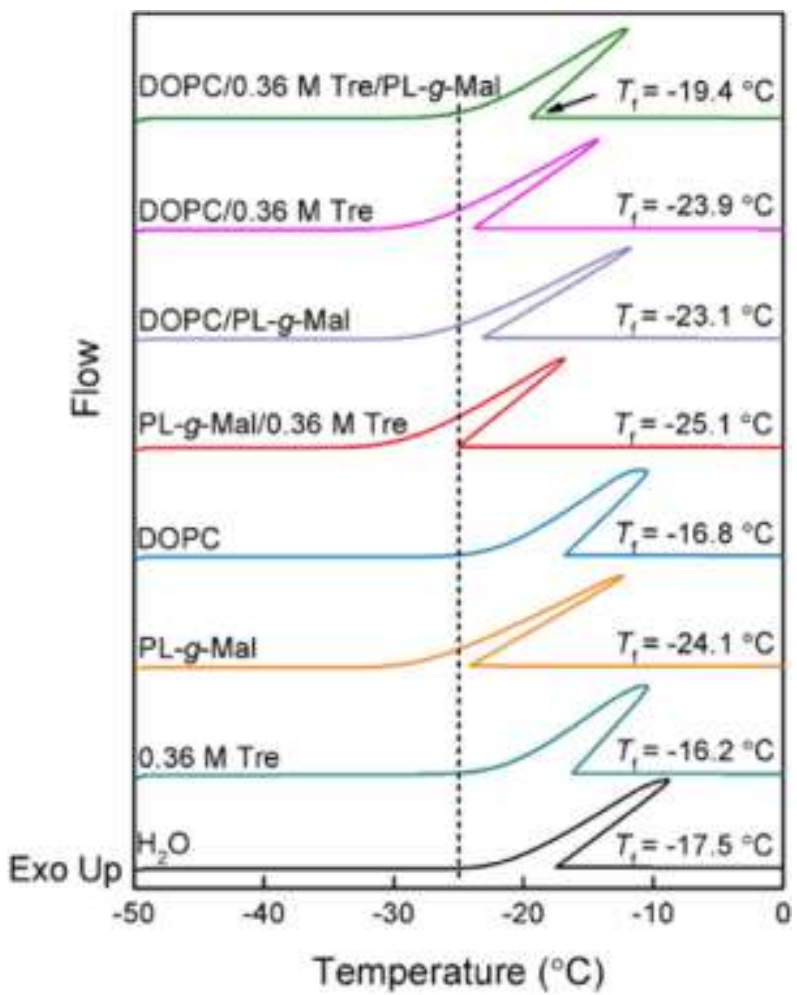

Figure S11. DSC cooling thermograms and freezing point $\left(T_{\mathrm{f}}\right)$ of $1 \mathrm{mM}$ DOPC, $0.36 \mathrm{M}$ trehalose, $1.0 \mathrm{mg} \mathrm{mL}^{-1} \mathrm{PL}-\mathrm{g}$-Mal and the corresponding mixture, respectively. The dotted line displayed the minimal $T_{\mathrm{f}}$ of PL-g-Mal/0.36 M Tre. 
(a)

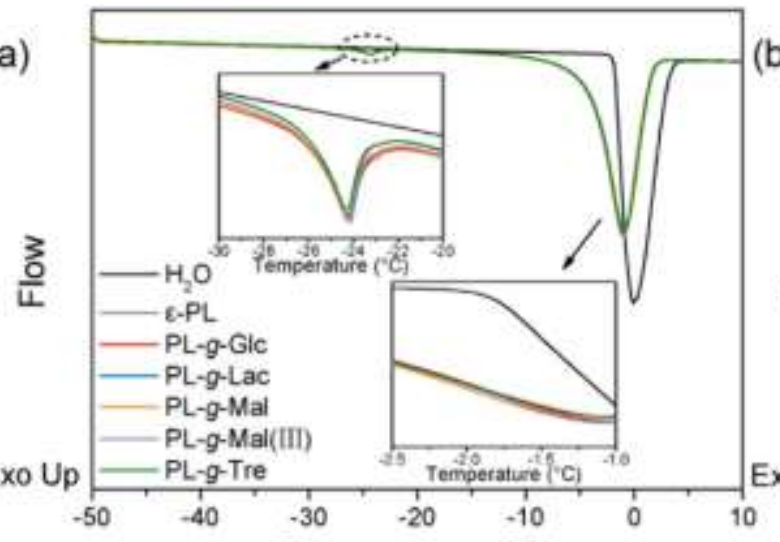

Temperature $\left({ }^{\circ} \mathrm{C}\right)$

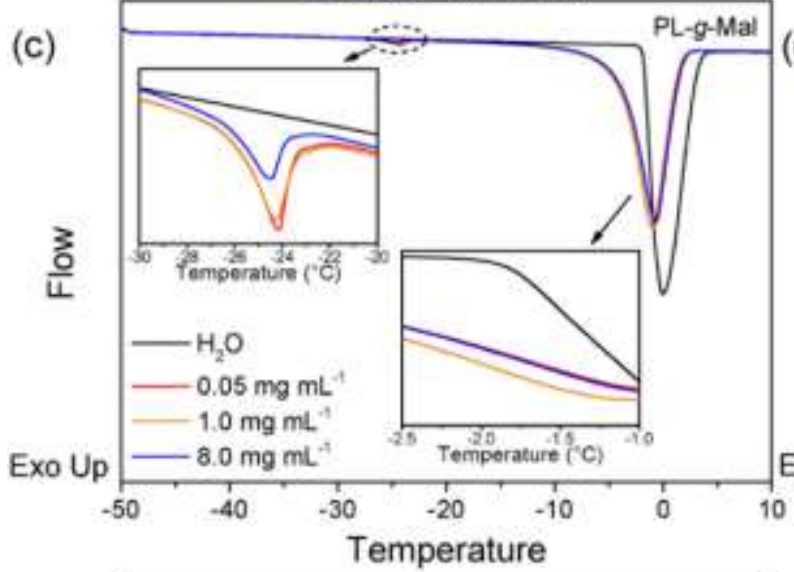

(e)

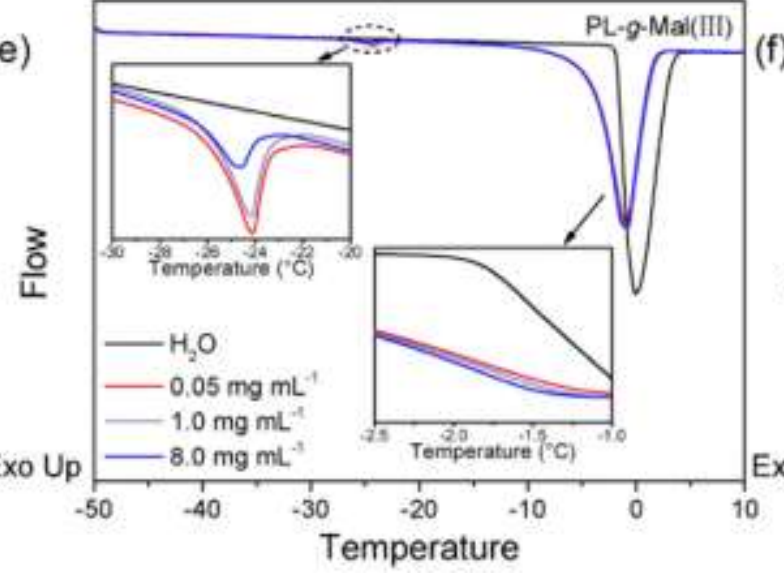

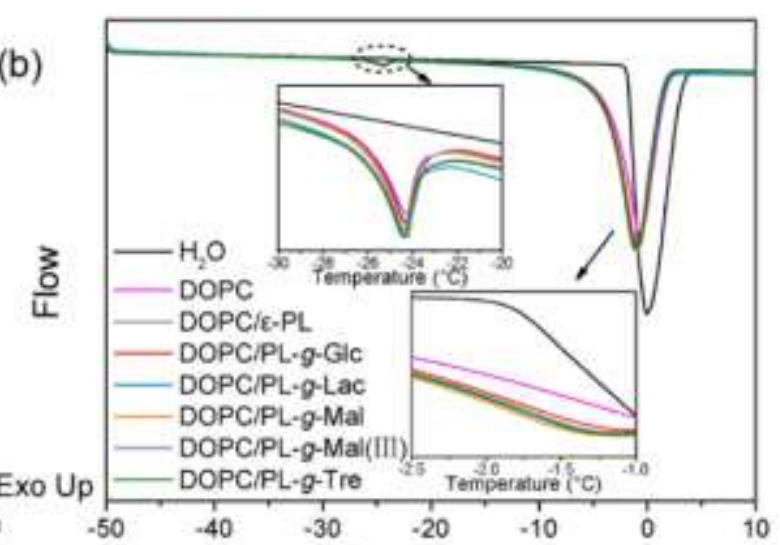

Temperature

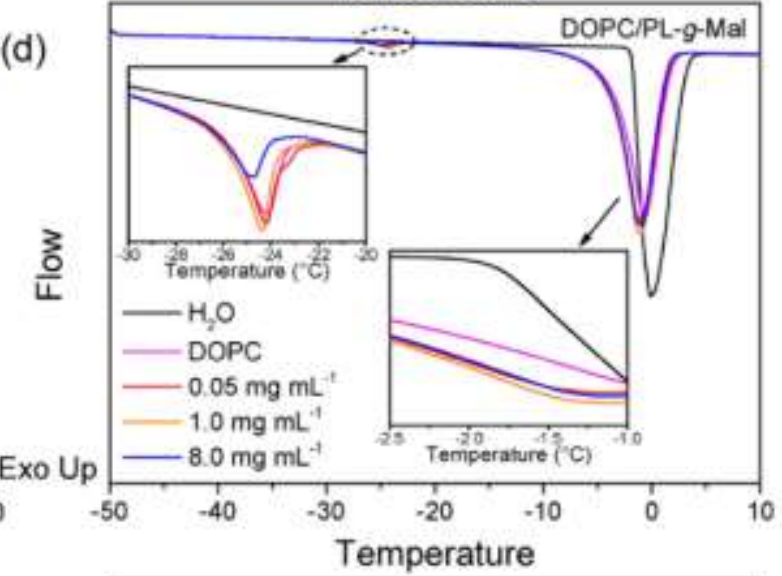

(f)

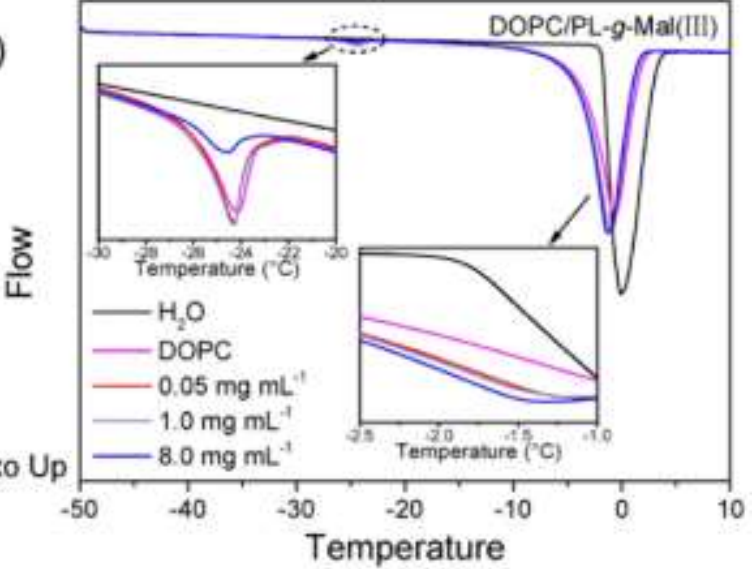

Figure S12. DSC heating thermograms of $1.0 \mathrm{mg} \mathrm{mL}^{-1}$ PL-g-S (a), PL-g-Mal (c) and PL-g-Mal(III) (e) in different concentrations as well as its corresponding mixture (b,d,f) with 1 mM DOPC, respectively. 


\section{Morphology of hRBCs.}
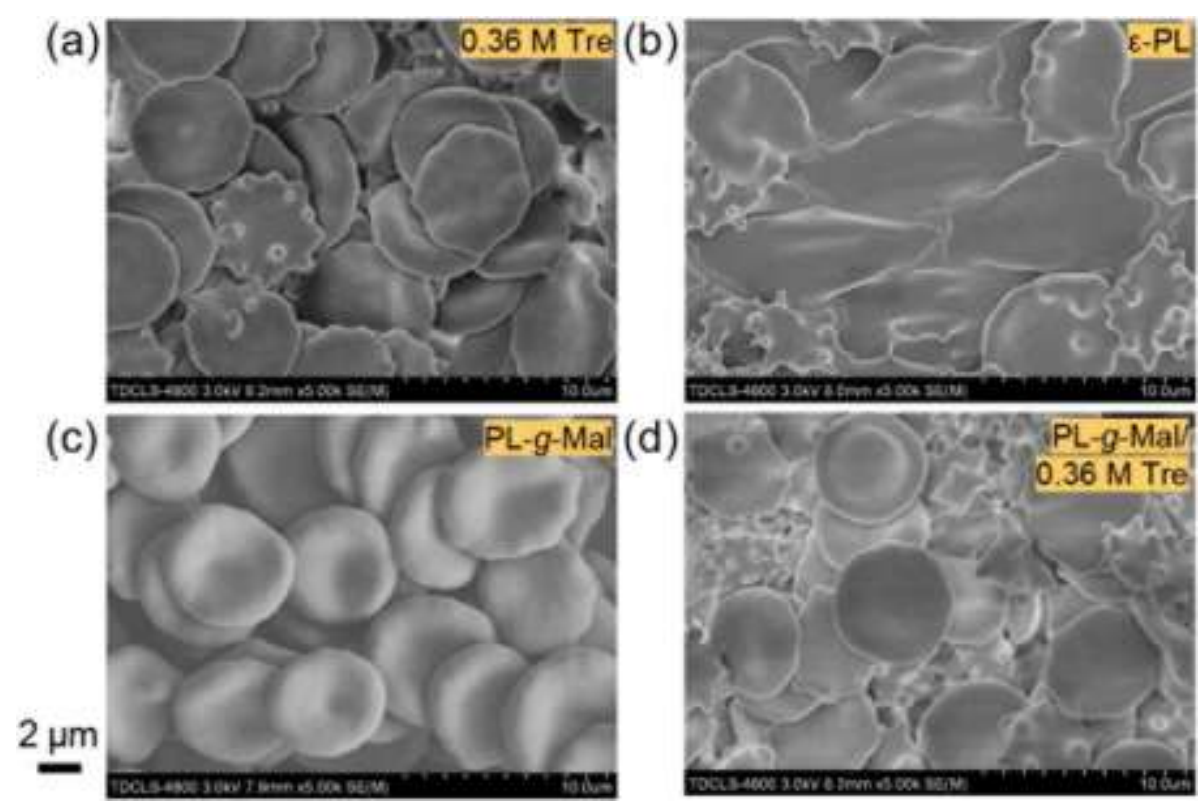

Figure S13. SEM images of hRBCs incubated by $0.36 \mathrm{M}$ trehalose (a), $\varepsilon-\mathrm{PL}$ (b), PL-g-Mal (c) and corresponding mixture (d) before $(a, b, c)$ and after $(d)$ cryopreservation. $(a, b)$ Without washing; (c,d) With 1× HEPES washing.

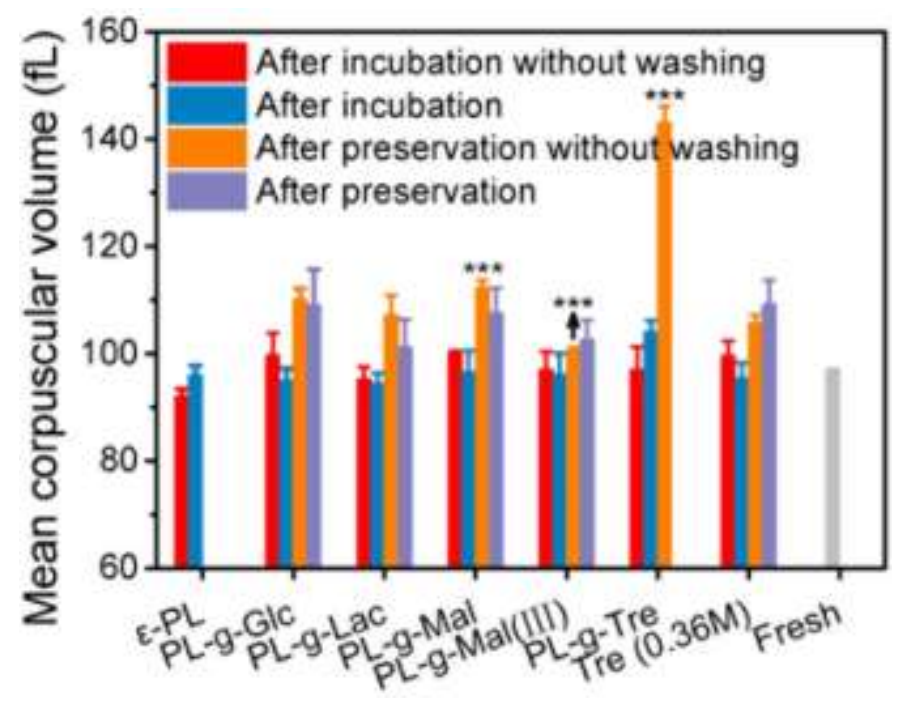

Figure S14. Mean corpuscular volume (MCV) of hRBCs before and after cryopreservation by PL- $g$-S along with $0.36 \mathrm{M}$ trehalose with or without washing. 


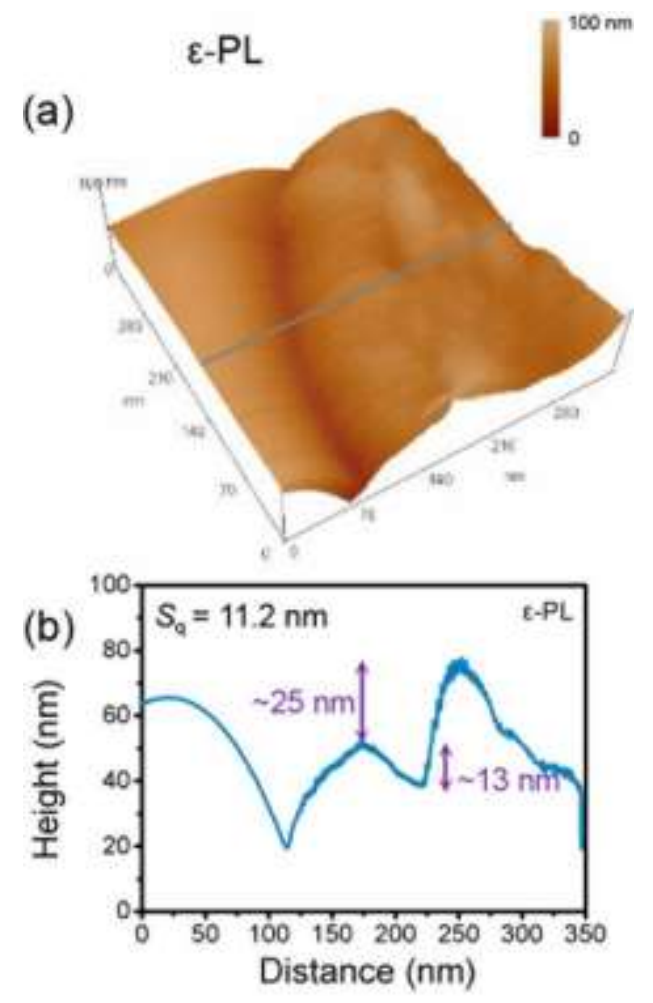

Figure S15. AFM images (a) and the corresponding line profiles (b) of human erythrocytes after incubation by $1.0 \mathrm{mg} \mathrm{mL}^{-1} \varepsilon-\mathrm{PL}$ without washing.

\section{REFERENCES}

(1) Lepoittevin, B.; Elhiri, A.; Bech, L.; Belleney, J.; Baltaze, J.-P.; Capron, I.; Planchot, V.;

Roger, P. Easy Access to Amphiphilic Glycosylated-Functionalized Polystyrenes. Carbohydr. Polym. 2011, 83, 1174-1179.

(2) Perouzel, E.; Jorgensen, M. R.; Keller, M.; Miller, A. D. Synthesis and Formulation of Neoglycolipids for the Functionalization of Liposomes and Lipoplexes. Bioconjug. Chem. 2003, $14,884-898$. 
(3) Debnath, K.; Pradhan, N.; Singh, B. K.; Jana, N. R.; Jana, N. R. Poly(trehalose) Nanoparticles Prevent Amyloid Aggregation and Suppress Polyglutamine Aggregation in a Huntington's Disease Model Mouse. ACS Appl. Mater. Interfaces 2017, 9, 24126-24139.

(4) Zhang, Q.; Liu, B.; Chong, J.; Ren, L.; Zhao, Y.; Yuan, X. Combination of Hydrophobically Modified $\gamma$-Poly(glutamic acid) and Trehalose Achieving High Cryosurvival of RBCs. Sci. China Technol. Sci. 2020, 64, 806-816.

(5) Liu, B.; Zhang, Q.; Zhao, Y.; Ren, L.; Yuan, X., Trehalose-Functional Glycopeptide Enhances Glycerol-Free Cryopreservation of Red Blood Cells. J. Mater. Chem. B 2019, 7, $5695-5703$.

(6) Chen, S.; Chen, R. A Virus-Mimicking, Endosomolytic Liposomal System for Efficient, pH-Triggered Intracellular Drug Delivery. ACS Appl. Mater. Interfaces 2016, 8, 22457-22467.

(7) Graham, B.; Fayter, A. E. R.; Houston, J. E.; Evans, R. C.; Gibson, M. I. Facially Amphipathic Glycopolymers Inhibit Ice Recrystallization. J. Am. Chem. Soc. 2018, 140, $5682-5685$.

(8) Hasan, M.; Fayter, A. E. R.; Gibson, M. I. Ice Recrystallization Inhibiting Polymers Enable Glycerol-Free Cryopreservation of Microorganisms. Biomacromolecules 2018, 19, $3371-3376$.

(9) Colina, J. R.; Suwalsky, M.; Manrique-Moreno, M.; Petit, K.; Aguilar, L. F.; Jemiola-Rzeminska, M.; Strzalka, K. Protective Effect of Epigallocatechin Gallate on Human Erythrocytes. Colloids Surf. B Biointerfaces 2019, 173, 742-750. 
(10) Deller, R. C.; Vatish, M.; Mitchell, D. A.; Gibson, M. I. Synthetic Polymers Enable Non-Vitreous Cellular Cryopreservation by Reducing Ice Crystal Growth during Thawing. Nat. Commun. 2014, 5, 3244-3250.

(11) Lee, S.; Lee, J. H.; Kim, H. W.; Hong, J. W. Properties of Phase Transition of Ice Binding Protein from Arctic Yeast (LeIBP) Utilizing Differential Scanning Calorimetry (DSC) and Raman Spectroscopy. Cryobiology 2018, 85, 33-38.

(12) Bai, S.; Li, X.; Zhang, R.; Li, C.; Zhu, K.; Sun, P.; Zhao, Y.; Ren, L.; Yuan, X. Enhancing Antifogging/Frost-Resisting Performances of Amphiphilic Coatings via Cationic, Zwitterionic or Anionic Polyelectrolytes. Chem. Eng. J. 2019, 357, 667-677.

(13) Yu, G.; Li, R.; Hubel, A. Interfacial Interactions of Sucrose during Cryopreservation Detected by Raman Spectroscopy. Langmuir 2019, 35, 7388-7395.

(14) Bai, G.; Song, Z.; Geng, H.; Gao, D.; Liu, K.; Wu, S.; Rao, W.; Guo, L.; Wang, J. Oxidized Quasi-Carbon Nitride Quantum Dots Inhibit Ice Growth. Adv. Mater. 2017, 29, 1606843.

(15) Geng, H.; Liu, X.; Shi, G.; Bai, G.; Ma, J.; Chen, J.; Wu, Z.; Song, Y.; Fang, H.; Wang, J. Graphene Oxide Restricts Growth and Recrystallization of Ice Crystals. Angew. Chem. Int. Ed. 2017, 56, 997-1001.

(16) Zhang, X.; Xu, X.; Li, Y.; Hu, C.; Zhang, Z.; Gu, Z. Virion-Like Membrane-Breaking Nanoparticles with Tumor-Activated Cell-and-Tissue Dual-Penetration Conquer Impermeable Cancer. Adv. Mater. 2018, 30, e1707240. 
(17) Butt, H. J.; Wolff, E. K.; Gould, S. A. C.; Northern, B. D.; Peterson, C. M.; Hansma, P. K. Imaging Cells with the Atomic Force Microscope, J. Struct. Biol. 1990, 105, 54-61.

(18) Zachee, P.; Snauwaert, J.; Vandenberghe, P.; Hellemans, L.; Boogaerts, M. Imaging Red Blood Cells with the Atomic Force Microscope. Br. J. Haematol. 1996, 95, 472-481. 